\title{
An unusual cause of small bowel obstruction
}

\author{
Sidhartha Sinha $\cdot$ Kezia Gaitskell $\cdot$ Rudy Borgstein • \\ Romi Navaratnam
}

Received: 29 June 2009/ Accepted: 23 September 2009/Published online: 5 November 2009

(C) SIMI 2009

\section{Case report}

A 95-year-old woman with a virgin abdomen presented to the Emergency Department (ED) with a 1 week history of vomiting, absolute constipation and generalised abdominal pain. Physical examination revealed tender abdominal distension with hyperactive bowel sounds and no hernias. Abdominal plain film radiograph (PFR) showed dilated small bowel loops without an obvious transition point or a radiologically apparent cause (Fig. 1a). Abdominal computed tomography scan (CT scan) revealed a cholecysto-duodenal fistula, pneumobilia and dilated small bowel loops proximal to a $1 \mathrm{~cm}$ diameter calcified intra-luminal structure in the distal ileum consistent with an ectopic gallstone (Fig. 1b, c). The patient underwent mini-laparotomy through a right iliac fossa incision, enterolithotomy and extraction of a $2.5 \mathrm{~cm}$ diameter gallstone (Fig. 1d), from which she recovered uneventfully.

\section{Discussion}

Gallstone ileus (GSI) accounts for 1-3\% of cases of small bowel obstruction (SBO) in the general population but is

S. Sinha $\cdot$ K. Gaitskell $\cdot$ R. Borgstein $\cdot$ R. Navaratnam

North Middlesex University Hospital NHS Trust, London, UK

S. Sinha $(\bowtie)$

27 Aylmer Road, London N2 0BS, UK

e-mail: sid261@ hotmail.com more common in those patients over the age of 65 where it is responsible for up to $25 \%$ of cases of non-strangulated SBO [1]. While PFR is likely to confirm the presence of dilated bowel, it is rarely useful in diagnosing GSI since only $10 \%$ of gallstones are calcified and thus visible as radio-opaque structures [2]. Ultrasound is purported to have greater than $95 \%$ specificity and sensitivity in detecting gallstones within the gallbladder, but is of limited use in GSI due to gaseous distension of the bowel [2]. A CT scan is more reliable than PFR in detecting the classical radiological manifestations of GSI described by Rigler's triad (pneumobilia, intestinal obstruction and visualization of a gallstone within the bowel), but it too will fail to detect a non-calcified gallstone $[1,2]$. Although gallstones with a diameter of less than $2.5 \mathrm{~cm}$ may pass spontaneously, a CT scan will not accurately identify the true size of a partially calcified gallstone (as in this case), and thus should not be used to determine the appropriateness of surgery.

Enterolithotomy alone is appropriate as primary treatment because remaining gallstones pass spontaneously without causing symptoms in $80-90 \%$ of cases and closure of the fistulous tract occurs spontaneously in the absence of persisting cholelithiasis. However, given that $10 \%$ of patients require reoperation for persistent biliary symptoms (such as recurrent gallstone ileus, cholecystitis and cholangitis) others advocate a definitive procedure (enterolithotomy, cholecystectomy and repair of biliary-enteric fistula), particularly in patients with minimal co-morbid conditions [1].

Bowel obstructions are responsible for $20 \%$ of surgical admissions with acute abdominal pain and $80 \%$ of these cases are due to small bowel pathology [3]. While the exact etiology of small bowel obstruction is often impossible to determine in the ED, it is prudent to involve the surgical service early in the care of such patients. 
Fig. 1 a Abdominal plain film radiograph showing dilated small bowel loops, $\mathbf{b}$ unenhanced CT scan showing gas within a thick-walled gallbladder (single arrowhead), duodenal cap (twin arrowheads) and cholecysto-duodenal fistula (arrow), c unenhanced CT scan showing calcified intra-luminal structure (arrow) with proximal dilated fluid-filled small bowel (twin arrowheads) and distal collapsed ileum (single arrowhead), $\mathbf{d}$ intra-operative photograph showing extraction of ectopic gallstone
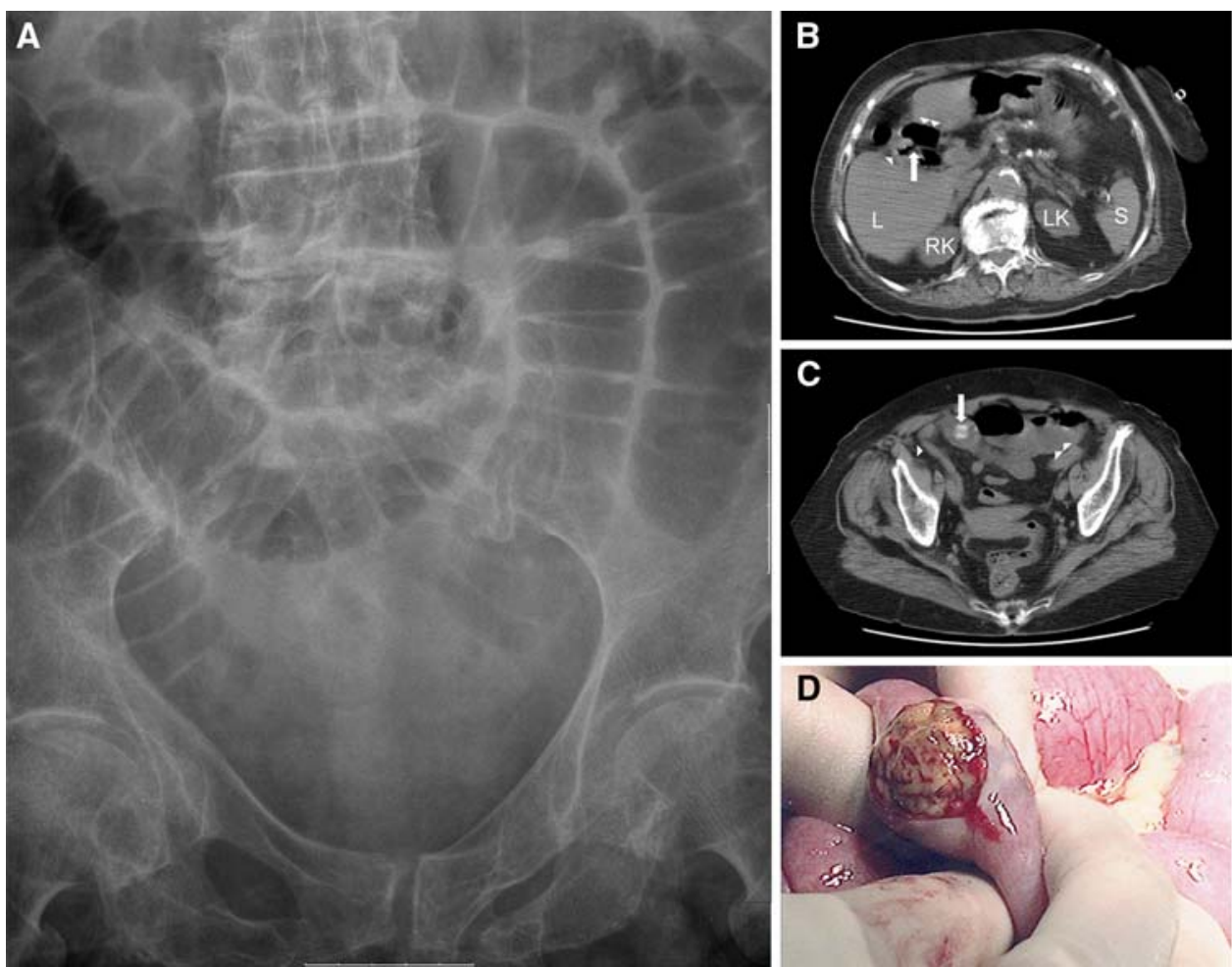

Conflict of interest statement The authors declare that they have no conflict of interest related to the publication of this manuscript.

\section{References}

1. Vagefi PA, Ferguson CM, Hall JF (2008) Recurrent gallstone ileus. Arch Surg 143(11):1118-1120
2. Portincasa P, Moschetta A, Petruzzelli M, Palasciano G, Di Ciaula A, Pezzolla A (2006) Symptoms and diagnosis of gallbladder stones. Best Pract Res Clin Gastroenterol 20(6):1017-1029

3. Attene J, Pepe G, Vanni S, Chiarlone M, Dilaghi B, Grifoni S (2009) A 64-year-old man admitted to the Emergency Department with an unusual case of intestinal obstruction: decision making in the Emergency Department. Intern Emerg Med 4:45-51 